tinuously to any height desired. It is sufficiently loud to be heard over a large lecture room, and is now so demonstrated.

It makes a very convincing experiment to produce the difference tone when all three tones are heard at once, and then gradually raise the pitch of the generating tones above the audibility limit, finally leaving the difference tone to be heard alone. The effect is by far the best that I have ever been able to obtain with 'tonvariators' of any kind. The method seems to be of no use in generating audible summation tones since, as in all methods, the necessary low tones mask the summational higher ones. It may be the case that this method of generating difference tones has been used by others though I have never seen a reference to it.

Prof. H. F. Batho has directed my attention to the fact that difference tones can also be produced by two radio-frequency generators similarly arranged, where the generating frequencies are of the order of a couple of thousand kilocycles per second. In this case the variable condenser which changes the frequency of one generator needs to have its capacity altered only a very small amount in order to cover the audible range. But more gradual tuning can be made by connecting a second small variable condenser in parallel with the first, so that a considerable movement of the plates of the second will be required to carry the pitch of the difference tone through the range of audibility. It is interesting to note that difference tones can be generated by two frequencies of any magnitude up to several million cycles per second.

\section{University of Manitoba, Winnipeg.}

Frank Aluen.

\section{Units for Degree of Vacuum}

Mr. TownsEnd's recent suggestion for a new unit for degree of vacuum based on the logarithm of the numerical value of the pressure in millimetres of mercury is to be welcomed. The new unit successfully removes the clumsy expressions of the present convention. It is also appropriate to adopt $1 \mathrm{~mm}$. mercury pressure as the reference-level for the new system with logarithm of a pressure ratio.

In defining the new unit there is, however, no need to have an analogy with the definition of the decibel in the method of measuring power ratios; the analogy should rather be with the definition of the bel, one decibel being one tenth of a bel, and being used for the sake of obtaining more convenient quantities in communication engineering problems. Thus the new pressure unit ('vacuum unit') should be defined so that a pressure of $a \mathrm{~mm}$. of mercury becomes $A=\log _{10} \frac{1}{a}=-\log _{10} a$ 'vacuum units'.

May I further be allowed to suggest that the name for the new unit might be "McLeod". A pressure of, say, $1 \times 10^{-5} \mathrm{~mm}$. $\mathrm{Hg}$ would thus read $5 \mathrm{McLeods}$, $1 \times 10^{-6} \mathrm{~mm}$. Hg would become 6 McLeods, and $3 \times 10^{-6} \mathrm{~mm}$. Hg would be equal to $6-\log _{10} 3=$ $5 \cdot 52$ MeL

University,

R. Fernberg.

Manchester.
I sHould like to comment on Mr. F. H. Townsend's' recent communication to this journal, in which he proposed a logarithmic scale of pressure for highvacuum work.

These new vacuum units provide us with a valuable method for measuring pump speeds. When a pump evacuating a closed vessel into which leakage is inappreciable-a case of great practical interest-is operating at constant speed by volume, the pressure in the vessel is falling exponentially towards zero. Thus the height of vacuum measured in Townsend's units is increasing linearly. I suggest, then, that the speed of a pump may be measured by the rate of increase of vacuum (in the new units) which it produces in such a closed volume of standard size. In fact, if the speed of the pump be $V$ volume units per second, it is $10 \mathrm{~V}$ vacuum units per volume unit per second, taking the volume of the standard vessel equal to the original unit. Thus the maximum speed of the pump referred to in Mr. Townsend's letter is 860 'vac.'/cu.m./hr. at 38 'vacs.'. An advantage of this method is that the calculation of how long it will take to exhaust a given vessel to a given vacuum affords rather less scope for arithmetical errors.

As regards naming the new unit, why not call it 'vacuum unit' and, as above, abbreviate it to 'vac.'?

Department of Applied Physics,

G. A. P. Wyulte.

University, Glasgow. May 13.

${ }^{1}$ Townsend, F. H., Nature, 155, 545 (1945).

\section{Hexachlorocyclohexane as an Insecticide}

Mr. E. L. TAYLOR has given ${ }^{1}$ an account of work on the acaricidal properties of a 'new' insecticide of the general formula $\mathrm{C}_{6} \mathrm{H}_{6} \mathrm{Cl}_{6}$ (hexachlorocyclohexane). This substance has been available in France for some years under the trade name of 'Aphtiria'. It would appear that a patent covering the method of preparation was taken out in France by M. Louis Gindraux in 1941. It has been used as an insecticide during the War in large quantities by the French Government with excellent results.

The disagreeable odour which is associated with hexachlorobenzene can be masked fairly easily, as the concentration of the active agent is rarely above 2 per cent when used as an insecticide. M. Gindraux (in a personal communication) believes that it is possible to prepare it free from an offensive smell and is engaged actively on the problem.

As most of the experiments which have been conducted have necessitated the use of organic solvents such as acetone, kerosene, etc., it should be noted that these are themselves active causers of dermatitis in man and that cases of skin involvement are common when used in industry.

It is, however, possible that a satisfactory cream will be produced containing an adequate concentration of hexachlorocyclohexane which can be used for the treatment of scabies in man.

$$
\text { L. B. Bourne }
$$
(Medical Officer).

A. C. Cossor, Ltd.,

Highbury Grove, London, N.5. May 25.

1 Nature, 155, 393 (1945). 